\title{
The Spontaneous Emulsification of Entrained Inclusions During Casting of High Aluminum Steels
}

\author{
AKALYA RAVIRAJ, NADIA KOURRA, MARK A. WILLIAMS, GERT ABBEL, \\ CLAIRE DAVIS, WOUTER TIEKINK, SEETHARAMAN SRIDHAR, \\ and STEPHEN SPOONER
}

\begin{abstract}
Mold slag entrainment during the continuous casting process presents a late stage source of non-metallic inclusions (NMI) with a high likelihood of ending up in the final product. The reaction between the entrained slag phase and surrounding liquid steel in the continuous casting mold affects the inclusion morphology and properties. However, there is a lack of information on the kinetics of the NMI-steel reaction. A novel approach, utilizing controlled synthetic inclusion/metal samples, has been developed to study the reactions between free inclusion-slag droplets and steel. The technique combines High-Temperature Confocal Scanning Laser Microscopy (HT-CSLM), X-ray Computed Tomography (XCT) and advanced electron microscopy techniques offering rapid controlled heating performance and extensive characterization of the samples. This method offers the ability to observe the size, shape and composition of an unconstrained reacting inclusion and to investigate the interface between the materials with respect to reaction time. This study interrogates a low aluminum steel $(0.04 \mathrm{wt}$ pct) and a high aluminum steel (1 wt pct) in contact with an inclusion-slag phase with a starting composition aligned to a typical mold slag. It was found that the reaction between silica and aluminum across the interface of the two phases provided a driving force for spontaneous emulsification to occur. Products of such emulsification will have a significant effect on the inclusion size distribution and potentially the prevalence of inclusion retention in molten steels solidifying in the continuous caster (for example if emulsified buoyancy forces are reduced to near zero) and hence in the subsequent solid product.
\end{abstract}

https://doi.org/10.1007/s11663-021-02091-z

(C) The Author(s) 2021

\section{INTRODUCTION}

THE cleanliness of liquid steel is defined by the amounts of dissolved unwanted impurities and precipitated unwanted non-metallic phases. ${ }^{[1,2]}$ Practices to control cleanliness in steel refining are critical for continuous improvement of the quality of the finished products and casting operations. ${ }^{[3]}$ Improving the cleanliness of the steel would mean a lower fraction of impurities in the final product. Dissolved impurities such

AKALYA RAVIRAJ, NADIA KOURRA, MARK A WILLIAMS, CLAIRE DAVIS, and STEPHEN SPOONER are with the AMMC, WMG, University of Warwick, Coventry, CV4 7AL, UK. Contact e-mail: a.raviraj@warwick.ac.uk GERT ABBEL, and WOUTER TIEKINK are with the Tata Steel, R\&D, Wenckebachstraat 1, 1951 JZ Velsen-Noord, The Netherlands. SEETHARAMAN SRIDHAR is with the Colorado School of Mines, 1500 Illinois St., Golden, CO 80401.

Manuscript received June 17, 2020; accepted January 20, 2021.

Article published online February 22, 2021. as oxygen, sulfur, phosphorus and hydrogen are removed either by transferring them into another phase such as slag or gas, or by allowing the impurities to form insoluble phases, referred to as inclusions, in the steel matrix which are ideally removed by their flotation and capture at surfaces (e.g., slag/metal interface) ${ }^{[4]}$ In the latter case, such insoluble oxide and sulfides have to be controlled in terms of size, number distribution and morphology such that they do not result in critical product defects or cause problems with process flow. ${ }^{[5]}$ Steel cleanliness is a relative term, which depends on the targeted product requirements and can be measured on a quantitative or qualitative basis. To quantify steel cleanliness, the volume of non-metallic inclusions in steel can be measured or a qualitative assessment can be defined based on the quality of the final product and its machinability, (taking into account the specific standards for evaluating steel cleanliness such as ASTM E45 18a). ${ }^{[6,7]}$ This form of assessment has to be reliable, repetitive and has to be useful when developing and modifying the steel manufacturing process, especially during the liquid metal steelmaking, refining, teeming and continuous casting processes. ${ }^{[8]}$ 
Stokes floatation is the principle method for calculating the velocity of a discrete particle rising through a fluid medium. Traditionally, variables of viscosity, particle size and density are fixed for a given inclusion type. However, if there is a reaction between the particle and fluid the resulting chemical change may lead to a change in local steel viscosity and inclusion density, which would need to be taken into account. Other factors when considering inclusion removal to an interface are physical phenomena such as film drainage ${ }^{[9]}$ which would affect whether an inclusion floats into the slag layer, interfacial turbulence or even spontaneous emulsification, ${ }^{[10]}$ which would change the particle size. Several studies have shown that mass transfer between two high temperature liquid reactants can cause a decrease in interfacial tension leading to the phenomenon known as spontaneous emulsification. ${ }^{[1,12]}$ Understanding the potential for these behaviors is critical for a better understanding of the kinetic conditions for steelmaking, including but not limited to both refining times and the critical variable of particle size when calculating flotation in the continuous caster.

Mold slags are molten oxides, and may sometimes contain fluorides to improve fluidity, which are used to control lubrication and heat transfer during the continuous casting process. In addition the mold slag is a pathway for absorption of inclusions or a potential chemical reaction reagent which can alter or break down inclusions. ${ }^{[13]}$ Mold slags contain high levels of silica and this presents a challenge when casting high aluminum steels due to the reactivity between the dissolved aluminum in the steel melt and the slag formed, (Eq. [1]). The reactivity of dissolved $\mathrm{Al}$ in the steel melt poses a considerable challenge during processing in terms of undesirable reactions between the melt and its environment and loss of relatively expensive alloy yield.

$$
4[\mathrm{Al}]+3\left(\mathrm{SiO}_{2}\right)_{(1)} \rightarrow 2\left(\mathrm{Al}_{2} \mathrm{O}_{3}\right)_{(1)}+3[\mathrm{Si}]
$$

where species in the [ ] parenthesis are in the metal phase and species in the ( ) parenthesis are in the slag/ inclusion phase.

The correct choice and use of mold slags for continuous casting of high aluminum steels is extremely important as there can be a strong reaction between aluminum in the liquid steel and silica in the mold slag which results in changes in the composition and properties of the mold slag, and hence its performance, in turn affecting the final slab quality. Yan et al., ${ }^{[14]}$ developed a mold slag for high aluminum nonmagnetic steels (1.5 to $2.5 \mathrm{wt}$ pet of $\mathrm{Al}$ ) where silica was partially replaced with alumina. Other compositions with different ratios of calcia and alumina for high aluminum TRIP steels ${ }^{[15]}$ or fluoride-free and low-fluoride lime-alumina-based fluxes ${ }^{[16]}$ have also been investigated. These slags were evaluated based on their viscosity, crystallization and effects on steel quality. Yan et al., ${ }^{[14]}$ concluded that a calcia/alumina mold slag with a ratio between 1.1 and 1.6 was ideal as it had a low viscosity, low crystallization temperature, and good heat transfer properties which is appropriate for casting high-aluminum steels.
Al is a major alloying element in novel and next generation steels such as TRIP and low density steels, which is added for both weight reduction and to aid phase transformation kinetics. Unwanted reactions between $\mathrm{Al}$ containing steel and $\mathrm{SiO}_{2}$ from the slag can result in changes in the steel chemistry (replacement of $\mathrm{Al}$ with $\mathrm{Si}$ ), resulting in the degradation of product quality due to variations in the phase stabilities or formation of Si-oxides on the surface of the as-cast slab. The latter affects the descaling effectiveness, secondary oxide formation and can affect the galvanizing process making it difficult to use these steels in commercial automotive parts. ${ }^{[17]} \mathrm{A}$ decrease in $\mathrm{SiO}_{2}$ in the mold slag will cause problems with process control in the continuous casting process. For example, a replacement of $\mathrm{SiO}_{2}$ by $\mathrm{Al}_{2} \mathrm{O}_{3}$ can cause unwanted changes in the slag viscosity which is critical for controlling lubrication at the strand and mold interface as well as slag crystallization which is critical for controlling the heat transfer and therefore the thickness of the solid strand.

The reaction between the inclusions (any inclusions arising from the mold slag) and the liquid steel in the continuous casting mold directly affects the inclusion behaviors such as its residence time and therefore potential removal rate. ${ }^{[18]}$ In this paper the interaction between mold slag inclusions and liquid steel, liquid steel of two compositions (low, 0.04 wt pct, and high, 1 wt pct, $\mathrm{Al}$ content), is considered to determine reaction kinetics and any changes in the inclusion particle size and morphology. The results have been used to estimate the residence time of inclusions in the molten steel in the continuous casting process.

As non-metallic mold slag inclusions are detrimental to nearly all grades of steel, which can result in losses of yield, the findings from this paper offer relevant extended insight to the wider steel grade production landscape. Steel manufacturing is an extremely energy intrusive process that also has a high carbon footprint, as such improving any aspect of the yield would have an enormous effect on sustainability due to the large tonnage of steel produced worldwide. The steels used in this study are representative of AHSS (Advanced High Strength Steel) including TRIP (Trans- formation Induced Plasticity) steel and DP (Dual Phase) steel, which have gained widespread attention for their low cost, high strength and reasonable ductility, giving timely pertinence to study the inclusion-steel behaviors for these systems.

\section{EXPERIMENTAL}

Combining the use of a High-Temperature Confocal Scanning Laser Microscope (HT-CSLM) and X-ray Computed Tomography (XCT) allows an inclusion that was fully immersed in the liquid steel to be located in a quenched sample and non-destructively characterized for morphology before subsequent destructive characterization. Specific details of the equipment used and previous example of combining the two methods for an alternative application can be found in the literature. ${ }^{[11,12,19]}$ This initial non-destructive examination is 
unique and key to unveiling the reaction phenomena and true inclusion morphology of the studied particles compared to historical studies in this area, which have typically relied on examining layers of mold slag on liquid steel. ${ }^{[8]}$ This method allows the inclusion to be studied within the steel matrix and its behavior without destructing either material.

In this study, a synthetic oxide particle (resembling mold slag chemistry), was placed inside a hole, packed with iron powder, in a steel alloy sample. The composite sample was then melted in an IR furnace in the HT-CLSM, whilst being continuously imaged with a U.V laser to confirm the point when the sample became liquid (in addition to temperature measurement). After controlled time intervals the samples were quenched allowing for time resolved characterization.

\section{A. Materials and Sample Preparation}

Pellet samples were machined by electrical discharge machining (EDM). The pellet samples, Figure 1, are cylinders of steel $(5 \mathrm{~mm}$ diameter and $6 \mathrm{~mm}$ high) that have a hole drilled into the top surface. To produce the artificial inclusion, mold powder particles are heated above their melting temperature at $1473 \mathrm{~K}$ on a graphite plate (non-wetting surface) to form spheres and sieved for particles between 350 and $450 \mu \mathrm{m}$ diameter. A mold slag particle is placed into a drilled hole in the steel, which is then back filled with electrolytic pure iron powder. A steel stopper of the same bulk pellet alloy was used to press the hole closed.

The composition of the steel, iron powder and the mold slag are shown in Tables I and II. The composition of the steel was measured using Spark OES and the composition of the slag and iron powder was measured using ICP. The mold powder being used is currently being used and is suitable for a wide range of strip products, therefore it is being used to investigate its reaction and effects for increased aluminum levels in the steel.

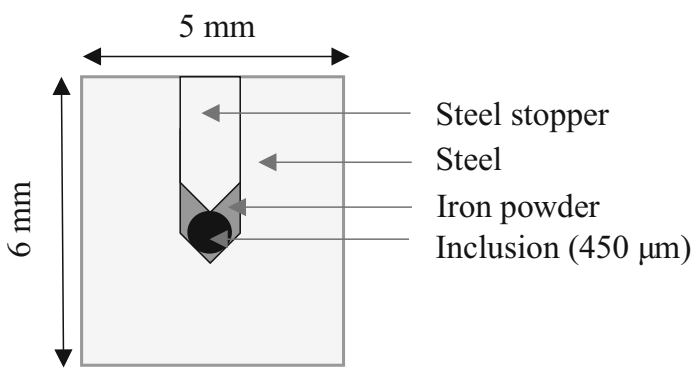

Fig. 1-Schematic of pellet sample showing the placement of the inclusion, the iron powder and the stopper.

\section{B. Experimental Procedures}

The sample was placed into an alumina crucible which was then loaded into the HT-CLSM. The chamber was vacuumed with a roughing pump and purged with high purity argon gas (99.9999 pct) three times to ensure a controlled atmosphere. The sample was preheated to $473 \mathrm{~K}$, using a slow heating rate, to ensure the longevity of the halogen lamp. The sample was then heated and quenched quickly with a heating regime of $500 \mathrm{~K}$ per minute from $473 \mathrm{~K}$ to $1893 \mathrm{~K}$ (the liquidus temperature of the steel is approximately $1803 \mathrm{~K}$ for 1 pct Al steel and this was used as a reference), held for a period of time ranging from 5 to 20 seconds. The sample was then quickly quenched at a rate of $1500 \mathrm{~K}$ per minute from $1893 \mathrm{~K}$ to $973 \mathrm{~K}$ and then cooled further at a rate of 300 $\mathrm{K}$ per minute to room temperature.

The quenched samples were then scanned in a Zeiss Versa 520 X-Ray CT scanner using a $160 \mathrm{kV}$ source, with an exposure time of 13 seconds and a magnification of $\times 0.4$. The resultant voxel size is 27 microns with 3235 projections. The data were analyzed using Avizo, a 3D visualization software that can segment the sample phases based on gray scale and examine features such as the size of the inclusion and any porosity. This can be done quantitatively given the known voxel size. ${ }^{[11]}$

After scanning the samples were mounted in resin using EpoThin ${ }^{\mathrm{TM}} 2$ Epoxy Resin and EpoThin ${ }^{\mathrm{TM}} 2$ Hardener. The samples were then sectioned (where the inclusion was identified from the XCT scan) and polished using a Buehler AutoMetTM 250 Pro Grinder-Polisher with a finishing diamond polish of 3 $\mu \mathrm{m}$. The SEM used for microscopic investigations was a Zeiss Supra 55VP and EDX was carried out on any inclusions present and the surrounding matrix.

\section{RESULTS}

Heat treatments in the HT-CLSM, under a gas environment that was inert with respect to the system, were used to enable a controlled reaction time between the liquid metal and slag phases under near isothermal conditions.

\section{A. Wt Pct Aluminum System}

Figure 2 shows XCT image slices from the 1 wt pct Al samples, obtained after heat treatment times of 5, 10 and 20 seconds. The results can be interpreted as the evolution of the inclusion as a function of time from 0 (a starting condition) to 20 seconds. The inclusion appears to break apart into many smaller particles (i.e.,

Table I. Composition of Iron Alloys and Powder Measured Using Spark OES and ICP Respectively, all Wt Pct

\begin{tabular}{llccccccccccccc}
\hline Element & $\mathrm{Fe}$ & $\mathrm{C}$ & $\mathrm{Si}$ & $\mathrm{Al}$ & $\mathrm{O}$ & $\mathrm{Mn}$ & $\mathrm{S}$ & $\mathrm{P}$ & $\mathrm{Ni}$ & $\mathrm{Cu}$ & $\mathrm{Cr}$ \\
\hline Low Al Steel & 99.7600 & 0.1974 & 0.0020 & 0.0400 & 0.0006 & - & - & - & - & - & - \\
High Al Steel & 98.800 & 0.1974 & 0.0020 & 1.0000 & 0.0006 & - & - & - & - & - \\
Iron Powder & 99.9968 & 0.0004 & 0.0001 & - & - & 0.0001 & 0.0010 & 0.0008 & 0.0002 & 0.0001 & 0.0002 \\
\hline
\end{tabular}


Table II. Composition of Mold Slag Powder was Measured Using ICP, all Wt Pct

\begin{tabular}{cccccccccccc}
\hline Compound & $\mathrm{Na}_{2} \mathrm{O}$ & $\mathrm{MgO}$ & $\mathrm{Al}_{2} \mathrm{O}_{3}$ & $\mathrm{SiO}_{2}$ & $\mathrm{SO}_{3}$ & $\mathrm{~K}_{2} \mathrm{O}$ & $\mathrm{CaO}$ & $\mathrm{MnO}$ & $\mathrm{FeO}$ & $\mathrm{F}$ \\
\hline & 3.5 & 1.1 & 7.4 & 42.2 & 0.3 & 0.1 & 41.6 & 0.2 & 0.5 & 3.3 \\
\hline
\end{tabular}

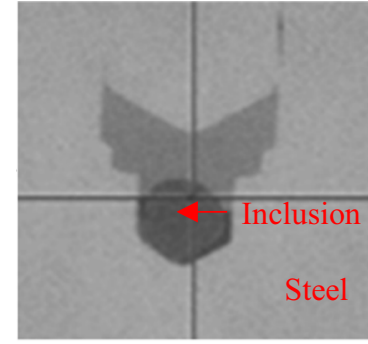

(a) Pre-heat treatment

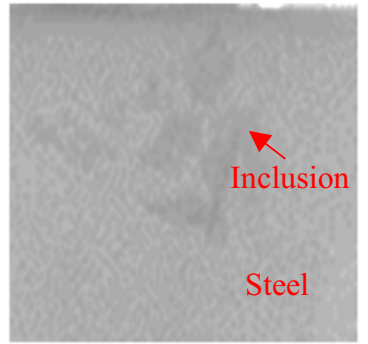

(b) $5 \mathrm{sec}$

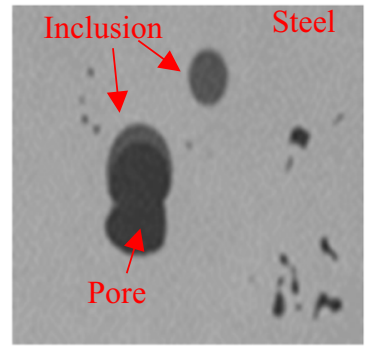

(c) $10 \mathrm{sec}$

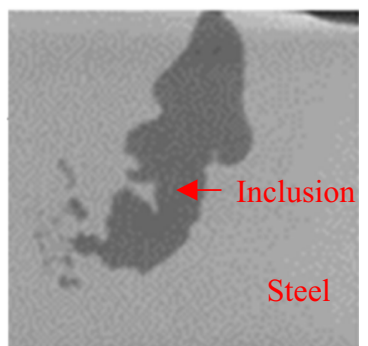

(d) $20 \mathrm{sec}$

Fig. 2-(a) to $(d)$ XCT slices showing the evolution of the inclusion immersed in steel as a function of time in 1 wt pct $\mathrm{Al}$ steels.

Table III. The Silica and Alumina Content in the Inclusion with Respect to Time (All Wt Pct)

\begin{tabular}{lrr}
\hline Time (s) & $\mathrm{SiO}_{2}$ & $\mathrm{Al}_{2} \mathrm{O}_{3}$ \\
\hline Pre-melting & 42.1 & 7.4 \\
5 & 17.9 & 31.7 \\
10 & 9.8 & 42.5 \\
20 & 2.8 & 46.8 \\
\hline
\end{tabular}

emulsify), clearly seen at 5 (Figure 2(b)) and 10 seconds (Figure 2(c)), and then start to recombine within a period of 20 seconds (Figure 2(d)) as the 20 seconds sample shows only a few small particles and one larger main inclusion.

The silica and alumina contents in the inclusion as a function of time from the quenched-sectioned samples are presented in Table III.

Previous work on the reaction between high aluminum steel and a silica rich slag ${ }^{[14,17]}$ has suggested that the kinetics follow a first-order relationship with aluminum diffusion being the rate controlling step through mass transfer in the metal phase. ${ }^{[20,21]}$

The XCT scan (Figure 2(b)) of the 5 second hold sample indicated that the inclusion had broken up into many small particles but the resolution of the scan was not sufficient to see this in detail, therefore the sample was sectioned in the area of interest and observed under SEM. The SEM image, as shown in Figure 3, shows the many small inclusion particles. EDS showed that these are inclusions with silica contents of an average of $17.9 \pm 1.5$ pct. EDS analysis of the steel in the area between the emulsified small inclusion particles shows that the aluminum content has reduced to $0.36 \mathrm{wt}$ pct, compared to the global aluminum content being still near to $1 \mathrm{wt}$ pet.
Figure 4 shows a 3D XCT reconstruction of the 10 second hold sample and SEM and EDS maps of the large inclusion obtained after sectioning the sample in the area of interest identified from the XCT scan. The EDS maps show the presence of silicon, aluminum and calcium in the oxide mixture. Further point analysis showed the silica content had reduced to $9.8 \mathrm{wt}$ pct and the alumina concentration had increased to $42.52 \mathrm{wt}$ pct. Using FactSage to compute the change in crystallization temperature of the inclusion as a result of this compositional change shows that the melting/solidification temperature increases from $1531 \mathrm{~K}$ (starting composition) to $1605 \mathrm{~K}$.

Figure 5(a) shows the 3D XCT reconstructed coalesced inclusion within the steel matrix at 20 seconds. Figure 5(b) is an SEM image of the inclusion. The silica content in this inclusion was found to have reduced to $2.8 \mathrm{wt}$ pct and the alumina content increased to $46.84 \mathrm{wt}$ pct. The inclusion is one particle at the top center of the sample, although the SEM image shows multiple particles due to a sectioning effect

The interfacial area between the metal and slag phases of samples treated for various times has been calculated using Avizo, a 3D visualization software, and is presented in Table IV.

\section{B. 0.04 Wt Pct Aluminum System}

A low aluminum steel (0.04 wt pct) was also investigated using the same test methods. The inclusion is seen to remain as one particle and not emulsify for each of the time steps investigated. This is shown in the XCT images in Figure 6. The corresponding silica and alumina wt pct of the inclusion as measured via SEM-EDS is given in Table $\mathrm{V}$. The composition is seen to stay relatively constant consistent with there being little reaction potential between the two phases in this system. 

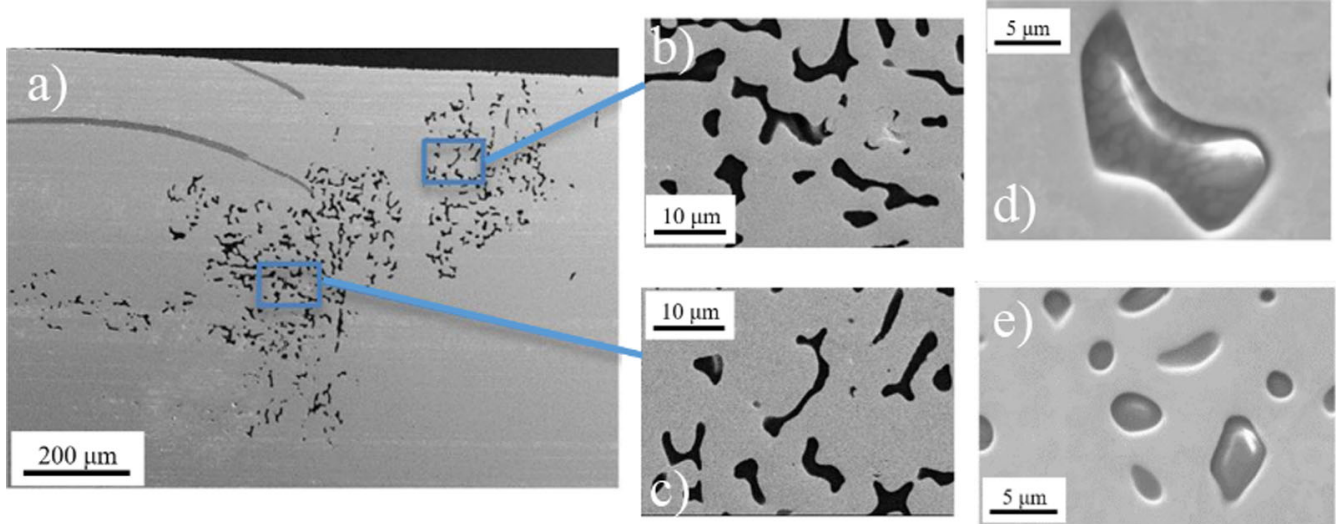

Fig. 3 - (a) $5 \mathrm{~s}$ hold emulsified sample under SEM with $(b)$ and $(c)$ at a higher resolution showing a clearer image of the shape of the inclusions, (d) an inclusion of approximately 10 micron size, (e) inclusions of approximately 5 micron size.

(a)

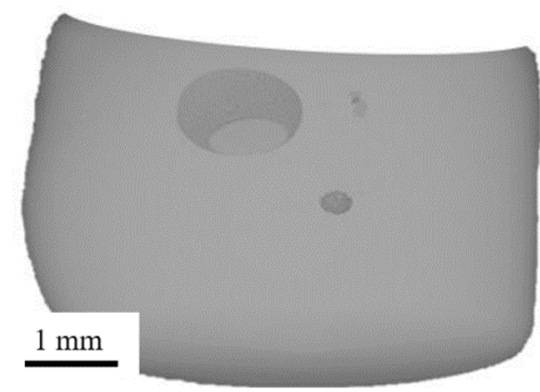

(b)

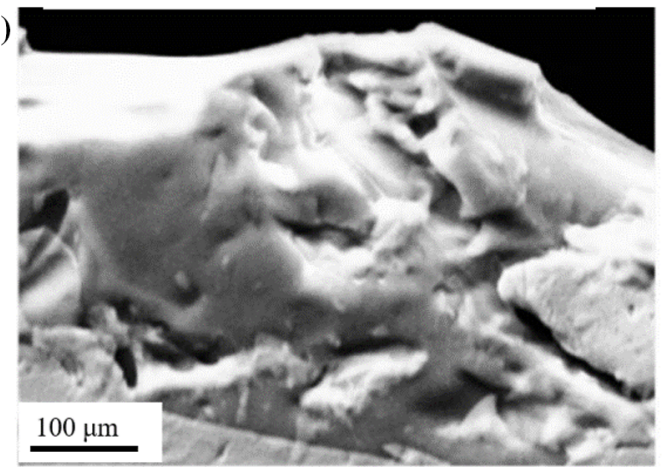

(c)
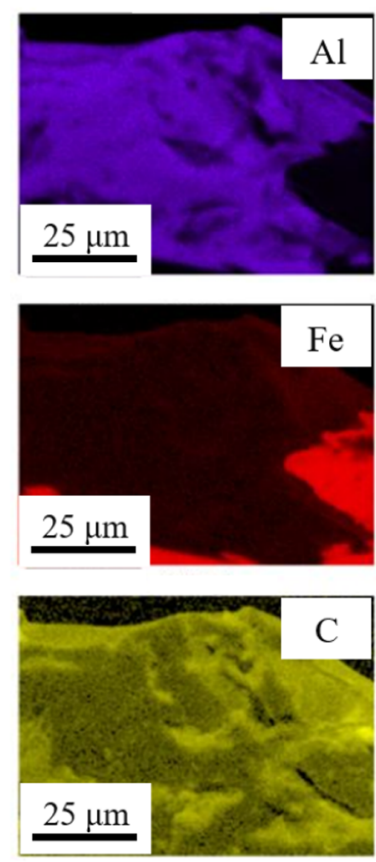
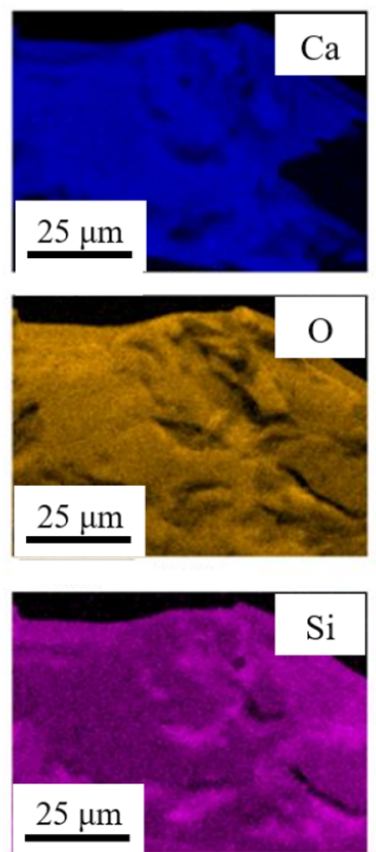

Fig. 4-The $10 \mathrm{~s}$ hold 1 pct sample $(a)$ 3D reconstruction of the sample from XCT data, $(b)$ SEM image of the inclusion area, $(c)$ map scans showing elemental composition of the area in view.

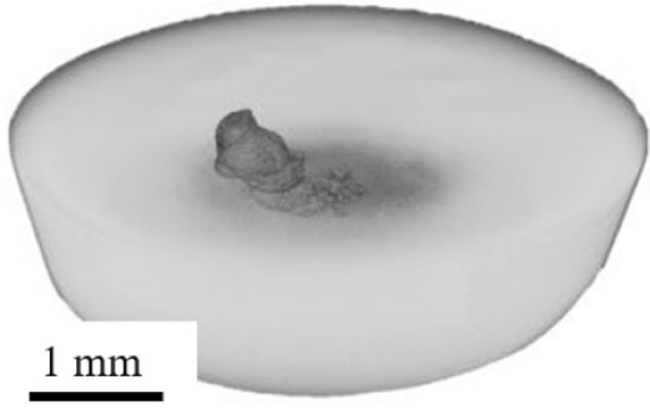

(a)

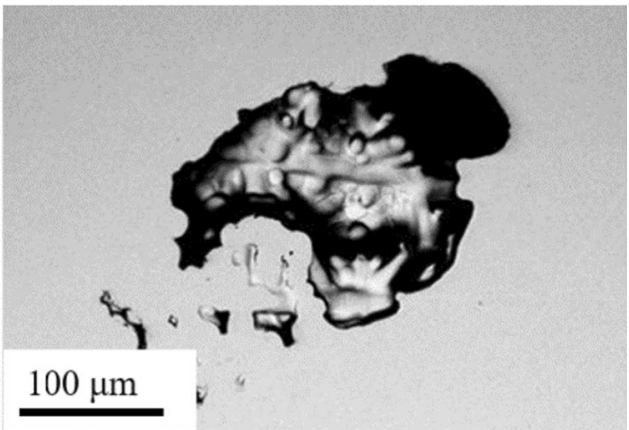

(b)

Fig. 5-20 s hold sample (a) 3D reconstruction of the sample showing the coalesced inclusion within the steel matrix, and (b) SEM image of the inclusion taken from the sectioned sample through the area of interest. 


\section{DISCUSSION}

\section{A. Why and How the Inclusion Emulsifies}

The reaction in Eq. [1] has a negative Gibbs free energy for both of the starting systems (high and low Al steels), so the forward reaction is favorable and there is a driving force for the reaction to proceed. Dynamic interfacial phenomena, i.e., spontaneous emulsification, was observed for the $1 \mathrm{wt}$ pct Al steel sample but not in the low-Al steel sample. A schematic of the progression of spontaneous emulsification, for the high Al-steel sample is shown in Figure 7 following the understanding presented previously in the literature for a high temperature mass transfer driven phenomenon occurrence. ${ }^{[19]}$

In the 0.04 pct $\mathrm{Al}$ samples, it is seen that the inclusion stays as one particle with little to no change in the inclusion chemistry. To form an emulsion, and to sustain an emulsion (increase in interfacial area), there needs to be an increase in material exchange between the steel and the inclusion. As the chemical potential in this system is much lower, it is not enough to form an emulsion - the energetic cost of increasing the interfacial area cannot be balanced by a greater negative energy force.

Spontaneous emulsification is triggered by gradients of chemical potential between the slag phase and metal phase. Emulsification occurs through a pathway of perturbation occurrence, growth, necking, and eventual budding of discrete particles away from the parent inclusion. The transient perturbations increase the interfacial area allowing for an increase in the reaction rate which further reduces interfacial tension, balancing

Table IV. Showing the Change in Surface Area of the Inclusion(s) with Respect to Time, with $A_{0}$ Being the Initial Surface Area and $A_{\mathrm{i}}$ Being the Surface Area at any Given Time Point

\begin{tabular}{lcc}
\hline Time $(\mathrm{s})$ & Surface Area $\left(\mathrm{mm}^{2}\right)$ & $A_{\mathrm{i}} / A_{0}$ \\
\hline Pre-melting & 0.6361 & 1.000 \\
5 & 8.445 & 13.27 \\
10 & 0.7854 & 1.234 \\
20 & 0.6648 & 1.045 \\
\hline
\end{tabular}

the energy cost and enabling further growth. The perturbations continue to grow until they reach a critical size upon which necking begins to occur due to fluid flow restriction down the perturbation. The neck narrows, where geometric strain causes this section of the perturbation to break off, causing budding, resulting in a lowering of system energy overall (even though area of interaction has increased). When an emulsion forms the global interfacial tension is at its highest, whereas the interfacial tension per unit area is at its lowest. The rate at which the Gibbs Free Energy decreases slows down as the chemical potential reduces. The emulsified particles begin to coalesce as the surface area is unstable as the interfacial tension increases and there is no longer a driving force for a highly sustained interfacial area due to the reducing chemical potential. The particle eventually coalesces to one particle which is spherical as its surface area and thus global interfacial tension is as low as possible, assuming that in this case, the emulsified small particles remain in close proximity.

The inclusion in the $1.0 \mathrm{wt}$ pct Al steel sample was emulsified after 5 seconds, which is shown in Figure 3, increasing the interfacial area by 13 times, and it is clear that spontaneous emulsification has taken place (as the conditions are the same as the 0.04 pct Al system apart from the composition of the metal) as the inclusion has broken apart into many smaller droplets. This is also accompanied by an initial rapid reduction in the silica content in the inclusion suggesting that a high reaction rate is associated with the emulsification. When the inclusion has emulsified, the local $\mathrm{Al}$ pct in the steel is $0.36 \mathrm{wt}$ pet compared to the global Al concentration which is still approximately $1 \mathrm{wt}$ pct. The reaction rate, manifested through the change in chemistry with time (Table IV) slows down, after which the particles start to

Table V. Showing the Composition of the Inclusion in the 0.04 Wt Pet Samples at 5, 60 and $300 \mathrm{~s}$

\begin{tabular}{lcc}
\hline Time (s) & $\mathrm{SiO}_{2}$ & $\mathrm{Al}_{2} \mathrm{O}_{3}$ \\
\hline 5 & 42.1 & 7.4 \\
60 & 41.8 & 7.2 \\
300 & 42.0 & 7.3 \\
\hline
\end{tabular}

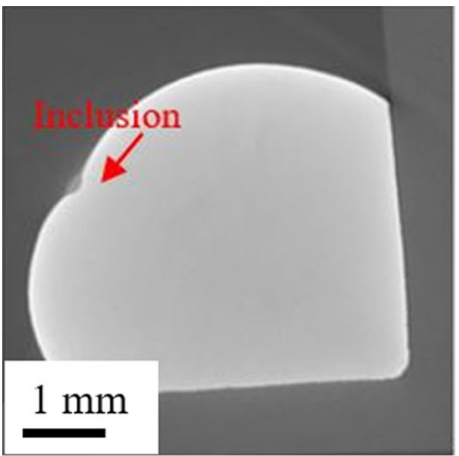

(a)

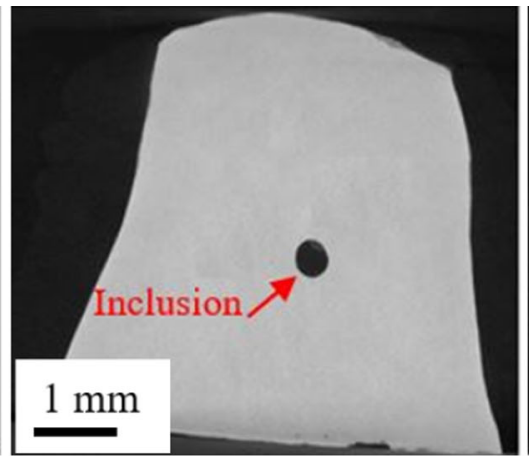

(b)

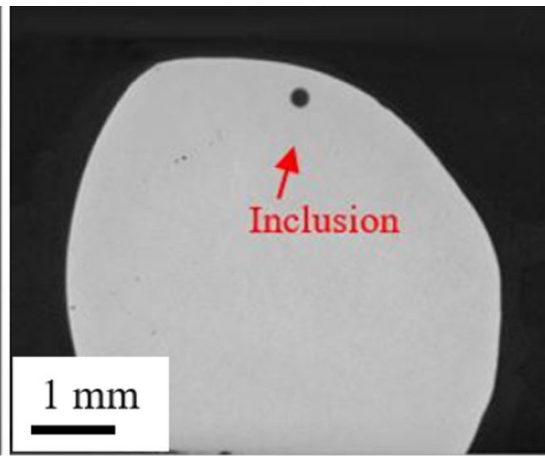

(c)

Fig. $6-0.04$ wt pct aluminium steels showing the inclusion to be one particle throughout the test (a) $5 \mathrm{~s},(b) 60 \mathrm{~s},(c) 300 \mathrm{~s}$. 

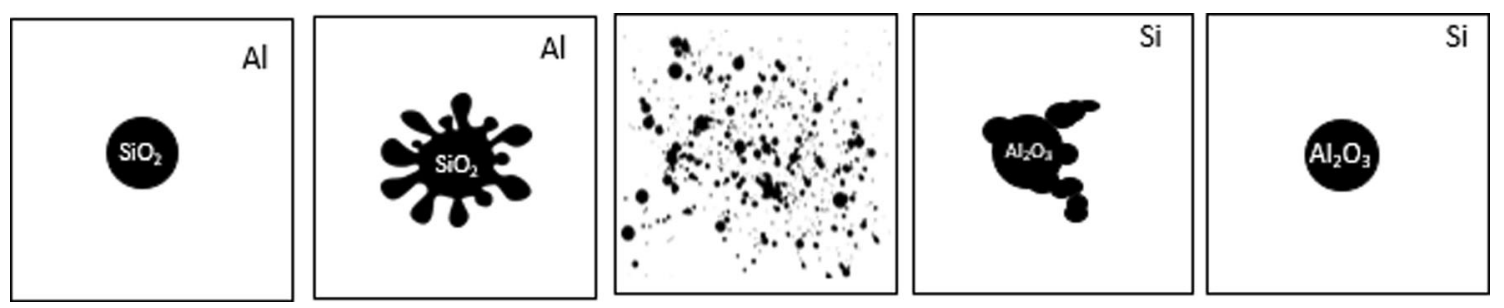

Fig. 7-Schematic of spontaneous emulsification with initial conditions of silica in the inclusion and aluminium in the steel.

coalesce. The theory of spontaneous emulsification states that the droplet should go back to a spherical shape as it is the lowest energy state ${ }^{[1,22]}$ but this is difficult to show in this case as it is hard to conduct the experiment to observe inclusions beyond about 20 to 25 seconds because of the size of the sample and the tendency of the inclusion to float to the surface. The driving force for spontaneous emulsification is the negative Gibbs free energy as a factor of time ${ }^{[22]}$ as well as the chemical potential of the reacting species balancing with other physical forces such as interfacial tension.

Many researchers have investigated the interfacial phenomena that occur between liquid metal and liquid slag during a reaction. Several studies have been conducted using $\mathrm{Fe}-\mathrm{Al}$ alloy droplets with $\mathrm{CaO}$ $\mathrm{SiO}_{2}-\mathrm{Al}_{2} \mathrm{O}_{3}$ slags. ${ }^{[22-25]}$ Spontaneous emulsification has been found to be immensely important as it can increase the rate of reaction significantly through the increase of interfacial area, thereby increasing the mass transport of the material by several orders. ${ }^{[26]}$

Metal droplets surrounded by slag were studied by Riboud and Lucas ${ }^{[27]}$ and it was found that the interfacial tension decreases drastically when a large enough mass transfer occurs at the interface. Ramadhani et al., ${ }^{[2]}$ have also studied reactions between slags and metal alloys with oxidizing elements. They found that the interfacial area when spontaneous emulsification occurs increases by 3 to 5 times of the original interfacial area, a surface area increase that is much lower.

Other authors have studied spontaneous emulsification in steel making due to the dephosphorization of a Fe-P drop immersed in a basic oxygen furnace type slag. The surface area of the metal drop is seen to rapidly increase by over one order of magnitude during the first 60 seconds of the reaction whilst the reaction is occurring at a fast rate. After 60 seconds when the reaction has slowed down, the droplets start to coalesce back together, minimizing the surface area and returning to a shape that is close to its equilibrium shape. ${ }^{[11,12]}$ It was reported that the surface area increased by ten times, a similar result to the system being investigated in this paper. Studies of metal droplets in slag have generally seen longer time scales for spontaneous emulsification to occur over than in the current study, which is the first reported study of liquid slag inclusions fully surrounded by liquid steel. It has previously been hypothesized that a physical restriction of factors such as viscosity and subsequent fluid flow may control the rate at which spontaneous emulsification can occur. The present study shows that with a high enough initial chemical potential the rate can be drastically increased despite having similar viscosities and phase properties to the previously referenced works.

\section{B. The Effect of Spontaneous Emulsification on Inclusion Behaviors}

Spontaneous emulsification could affect inclusion velocity as smaller inclusions will float slower than larger inclusions. Using Stokes floatation as a benchmark calculation, a 450 micron inclusion that is silica rich (such as one retaining the starting mold powder composition), would float at a speed of $0.167 \mathrm{~m} / \mathrm{s}$. As the reaction progresses, and an emulsion forms due to the decrease in interfacial tension, inclusions of a 10 micron size would float at $7.33 \times 10^{-5} \mathrm{~m} / \mathrm{s}$, reducing the floatation velocity significantly to a point where it is almost negligible. As the inclusion particle coalesces, and the inclusion becomes alumina rich, the velocity of the reported inclusion in Figure 5(a)) would be 0.149 $\mathrm{m} / \mathrm{s}$. These dynamic changes will affect inclusion floatation in the continuous caster ${ }^{[28]}$ as shown schematically in Figure 8. Assuming Stoke's floatation in a continuous caster where a mold flux inclusion is entrapped at the top of the continuous cast mold, giving a flotation distance of 30 meters for inclusion removal to the slag layer then an inclusion that is silica rich of 450 micron initial size would take 180 seconds to float, however, an inclusion that has reacted that is now alumina rich of the same size would take 201 seconds to float, which is an increase of 11.7 pct. Taking the reaction into account giving a short time frame where an emulsion exists, an inclusion that undergoes these dynamic changes would take 203 seconds to float, which is an increase of 12.7 pct. However coalescence rates in the continuous caster are expected to be less than that seen in the HT-CLSM experiment (due to fluid flow and turbulence) and these effects could be greatly increase the flotation time.

The time it takes to solidify steel fully depends on the process - in a thin slab caster it is approximately 2 minutes and in a thick slab caster it is approximately seventeen minutes, both of which are much longer than the reaction time that has been found (spontaneous emulsification occurring within 5 seconds). As a steel slab solidifies from the outside in, it can be assumed that an entrained slag particle entrapped near the surface of 


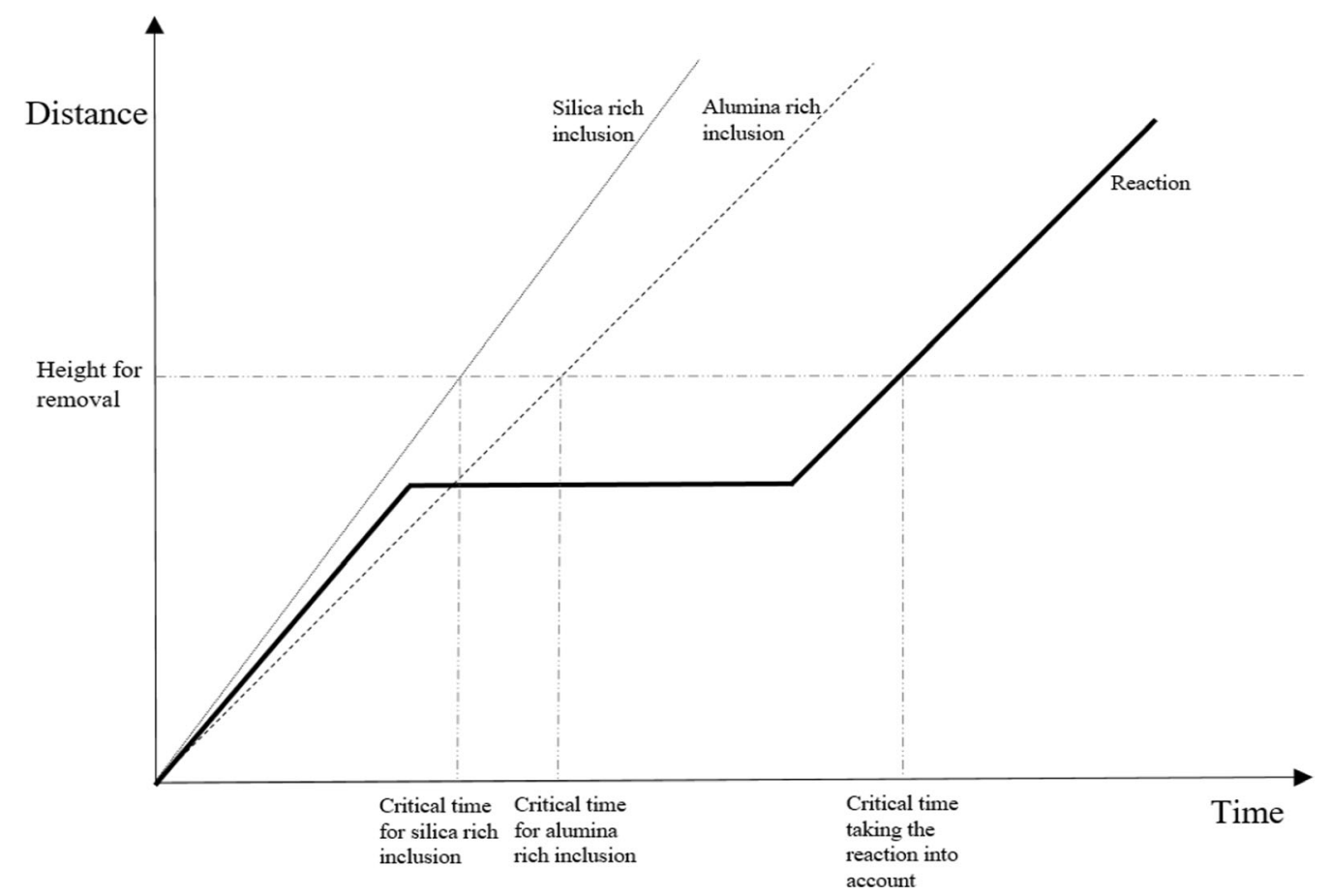

Fig. 8- Showing the critical time for silica rich and alumina rich inclusion to float and how the floatation could be affected if the inclusion undergoes spontaneous emulsification.

the slab has much shorter residence time (minimum time of a few seconds based on a flow speed of approximately $30 \mathrm{~cm} / \mathrm{s}$ ), whereas a particle that is in the middle of the slab (where the steel is liquid) could undergo greater levels of reaction. However, other phenomena such as film drainage, convection and steel flow would greatly affect the residence time of an inclusion and these factors are known to have large contributing effect on the overall modelling and understanding of the continuous caster. ${ }^{[9,29,30]}$

The advantages of note are that in the system being investigated, the inclusion is unbound, so interfacial tensions are unaffected by wetting to a third phase. Furthermore, non-destructive characterization for raw geometry detection and direct segmentation is enabled to allow further advanced characterization to be used to study the varying chemical compositions as a factor of time. Spontaneous emulsification would undoubtedly affect inclusion floatation in the continuous caster as smaller inclusions have less buoyancy than larger inclusions. As such the composition matching of more alloyed steel grades requires careful selection of the materials used in conjunction with their production.

The inclusions at each reaction time for the $1 \mathrm{wt}$ pct Al steel were analyzed and the chemical composition changes of the inclusion during the reaction were determined. Figure 9 shows the changes in $\mathrm{SiO}_{2}$ and $\mathrm{Al}_{2} \mathrm{O}_{3}$ during the reaction between the steel and inclusion at $1893 \mathrm{~K}$ in graphical output as opposed to the raw numbers presented in Table IV. The $\mathrm{SiO}_{2}$ content clearly decreased while the $\mathrm{Al}_{2} \mathrm{O}_{3}$ content increased suggesting that the reaction from equation 1 took place to a significant level.

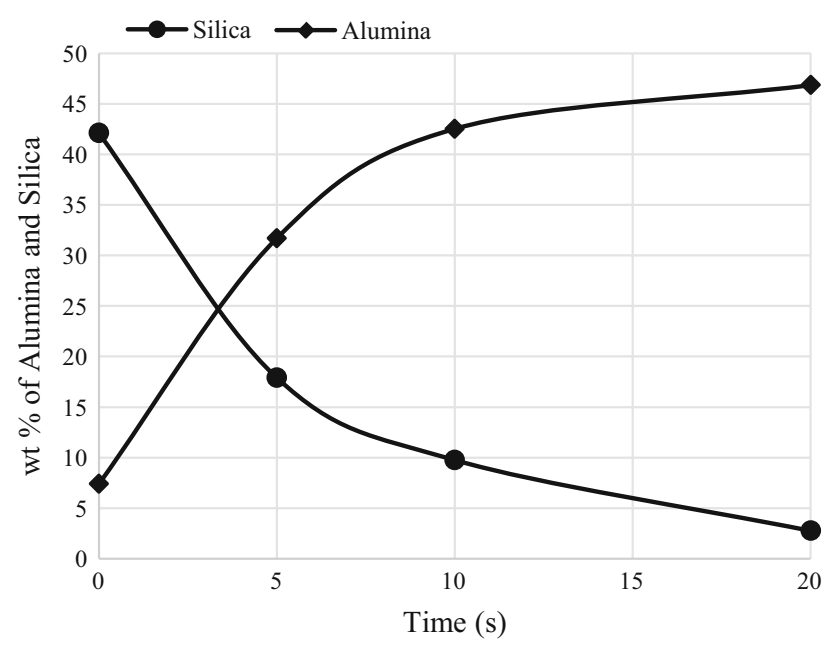

Fig. 9-The change of alumina and silica content in the inclusion during the reaction.

Figure 10 shows the changes in $\mathrm{SiO}_{2}$ and $\mathrm{Al}_{2} \mathrm{O}_{3}$ with relation to the changes in surface area of the inclusion over time. As the reaction progresses, there is a decrease in the driving force of the reaction as the chemical potential reduces, leading to the rate of reaction reducing. This would also indicate that the mass transfer in the slag phase has also decreased due to diminishing reactants, further shown by the rate of reaction reducing as seen in Figure 11. The critical rate of reaction was found to be $-0.0471 \mathrm{~mol} \mathrm{~s}^{-1}$ to sustain spontaneous emulsification. 


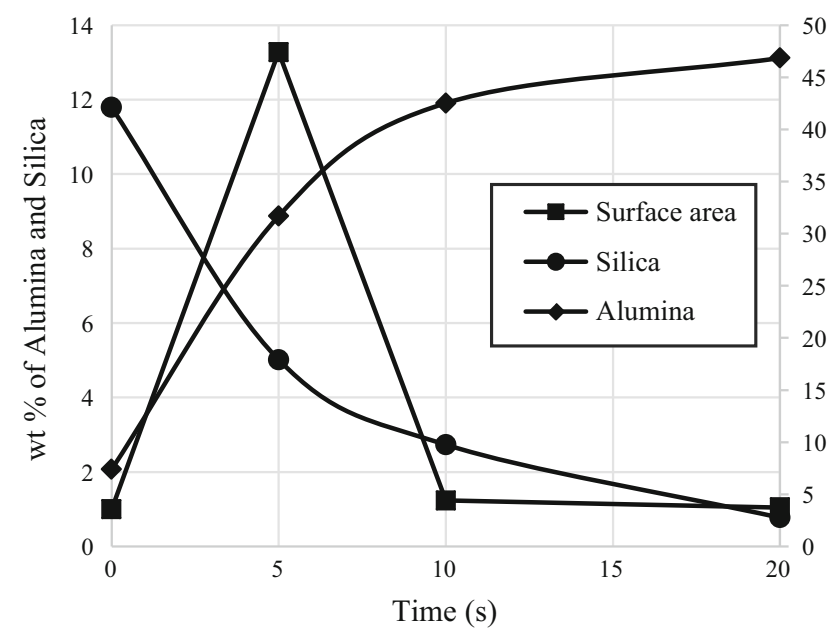

Fig. 10-The change of alumina and silica content in the inclusion during the reaction with the change in surface area.

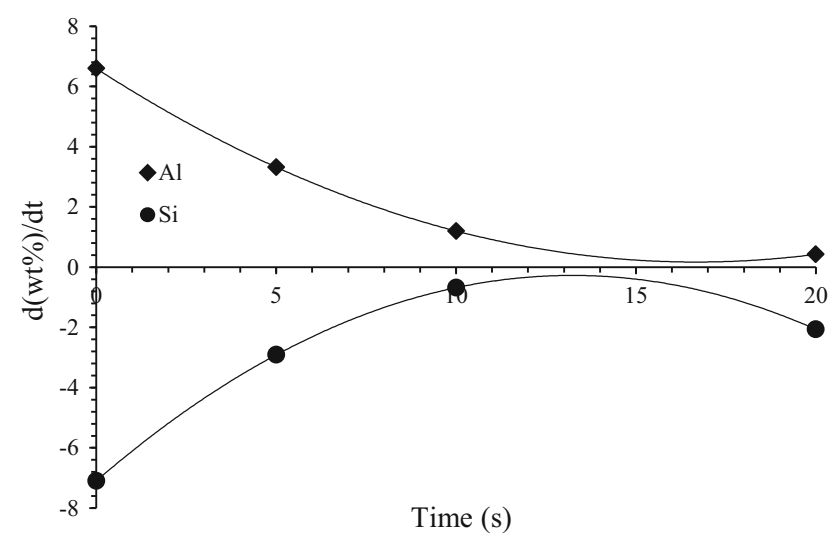

Fig. 11-Differential weight pct as a factor of time.

\section{Additional Inclusion Composition Change Effects in the Continuous Caster}

The agglomeration of inclusions has seen significant studies with regards to features such as aided buoyancy and submerged entry nozzle clogging. Findings have shown a range of conditions where inclusions don't/are less likely to coalesce, or where they have long range attractive natures. The results in this system indicates agglomeration of liquid inclusions. Hongbin et al. ${ }^{[31,32]}$ investigated the collision and agglomeration of inclusions in $\mathrm{CaO}-\mathrm{Al}_{2} \mathrm{O}_{3}$ and $\mathrm{CaO}-\mathrm{Al}_{2} \mathrm{O}_{3}-\mathrm{SiO}_{2}$ systems including solid or liquid as well as solid plus liquid on various steel melt surfaces with a HT-CSLM. They found that the capillary effect is shown to be responsible for the long-range attraction on a molten steel surface between two inclusion particles. The contact angle between a solid particle and steel melt, particle size and density as well as the surface tension of the steel melt influence the capillary attraction force. They also concluded that the attraction for solid $\mathrm{Al}_{2} \mathrm{O}_{3}-\mathrm{SiO}_{2}$ is weaker than that of pure solid alumina particles. It was also noted that in industrial practices of injection metallurgy, the long-range strong capillary attraction present between solid alumina particles on the surface of gas bubbles in the molten steel bath can result in quick collision, agglomeration and formation of alumina clusters in the steel bath. They observed liquid inclusions merging on the steel melt surface and they expect it to be similar to the steel bath due to physical features such as film drainage. Larger particles would overcome the drainage due to the stronger attraction force operating in between.

In addition to the possible coalescence of liquid inclusions with given conditions, there is a high probability of heterogeneous composition dispersion within the inclusions of this study. Given the described pathway of perturbation growth and eventual budding being the pathway of spontaneous emulsification, the entire phenomenon is reliant on spatially variable conditions. As the reaction being too slow, this is highly likely to result in enrichment of alumina within the inclusion at the head of a perturbation, which may go as far as to enable solidification of a localized portion of the inclusion for a general scenario. This would offer a pathway to greatly enable the agglomeration/coalescence of the clouded inclusion emulsion to begin the coalescent behaviors observed in the later stages of the inclusion lifecycle presented above.

\section{CONCLUSION}

To conclude, a low aluminum steel (0.04 wt pct) and a high aluminum steel (1 wt pct) in contact with a slag phase inclusion with oxide compositions that resemble a mold slag was used to interrogate the reaction between the aluminum in the steel and the silica in the slag, and the consequent effect on the inclusion size/shape.

Firstly, in the $1 \mathrm{wt}$ pct Al steel, the inclusion reacted with the Al (silica being replaced by alumina in the inclusion), forming an emulsion after 5 seconds, beginning to coalesce at 10 seconds, to reform one particle by 20 seconds. However, this behavior was not observed in the $0.04 \mathrm{wt}$ pct Al steel.

Secondly, the phenomena of spontaneous emulsification is seen to take place with the inclusion breaking apart into many small, typically 5 to 10 micron, inclusions, and coalescing to one particle again as the reaction with the $\mathrm{Al}$ in the liquid steel continued. This is due to the reaction having a high enough chemical potential to offer the driving force for emulsification to occur initially. This phenomena is supported with chemical composition measurements aligning with the lifecycle of the inclusion according to previously documented pathways of reaction driven spontaneous emulsification. A critical reaction rate of $-0.0471 \mathrm{~mol} \mathrm{~s}^{-1}$ was found to be needed for this system to cause and sustain spontaneous emulsification.

Thirdly, spontaneous emulsification of inclusions would increase the critical time for inclusion removal which would greatly affect the inclusion behaviors in the continuous casting mold. Times could rise from traditionally calculated residence periods of 180 seconds for a 450 micron inclusion, to potentially 203 seconds for an inclusion that follows the same break up and 
coalescence times reported here and compositional changes. As an inclusion in a continuous caster would be unbound, the statistical chance of recombination of the inclusion would be much lower and residence times extended even more.

Finally, due to the heterogeneous nature spontaneous emulsification occurs by the inclusion upon reaching reaction/emulsification equilibrium is unlikely to be completely homogenous. As such oversaturation of alumina at given points are likely to cause solidification and offer a lower energetic pathway to which emulsion coalescence may occur by.

\section{ACKNOWLEDGMENTS}

I would like to thank EPSRC and Tata Steel Europe for funding this research via the EngD scheme, to WMG for the provision of facilities and to Tata Steel Europe for providing the samples used in this study.

\section{OPEN ACCESS}

This article is licensed under a Creative Commons Attribution 4.0 International License, which permits use, sharing, adaptation, distribution and reproduction in any medium or format, as long as you give appropriate credit to the original author(s) and the source, provide a link to the Creative Commons licence, and indicate if changes were made. The images or other third party material in this article are included in the article's Creative Commons licence, unless indicated otherwise in a credit line to the material. If material is not included in the article's Creative Commons licence and your intended use is not permitted by statutory regulation or exceeds the permitted use, you will need to obtain permission directly from the copyright holder. To view a copy of this licence, visit http://creativecommons.org/licenses/by/4.0/.

\section{REFERENCES}

1. L. Zhang and B.G. Thomas: Proc. XXIV Natl. Steelmak. Symp., 2003, pp. 138-83.

2. E.B. Pretorius, H.G. Oltmann, and B.T. Schart: in Iron and Steel Technology, 2015.

3. L. Zhang, J. Aoki, and B.G. Thomas: Metall. Mater. Trans. B, 2006, vol. 37B, pp. 361-79.

4. R.J. Fruehan: Making, Shap. Treat. Steel-Steelmak. Refin. 1998, pp. 1-12.
5. L.F. Zhang and B.G. Thomas: ISIJ Int., 2003, vol. 43, pp. 271-91.

6. R.V. Väinölä, L.E.K. Holappa, and P.H.J. Karvonen: J. Mater. Process. Tech., 1995, vol. 53, pp. 453-65.

7. J. Yang, X.H. Wang, M. Jiang, and W.J. Wang: J. Iron Steel Res. Int., 2011, vol. 18, pp. 8-14.

8. L. Zhang, B.G. Thomas, X. Wang, and K. Cai: 85th Steelmak. Conf. ISS-AIME, 2002, pp. 431-52.

9. G. Shannon, L. White, and S. Sridhar: Mater. Sci. Eng. A, 2008, vol. 495 , pp. $310-15$.

10. Y. Chung and A.W. Cramb: Philos. Trans. R. Soc. Lond. Ser. A, 1998, vol. 356, pp. 981-93.

11. A.N. Assis, J. Warnett, S. Spooner, R.J. Fruehan, M.A. Williams, and S. Sridhar: Metall. Mater. Trans. B., 2015, vol. 46B, pp. 56876.

12. S. Spooner, A.N. Assis, J. Warnett, R. Fruehan, M.A. Williams, and S. Sridhar: Metall. Mater. Trans. B., 2016, vol. 47B, pp. 212332.

13. Z.Q. Liu, F.S. Qi, B.K. Li, and S.C.P. Cheung: Int. J. Multiph. Flow, 2016, vol. 79, pp. 190-201.

14. M. Yan, A. McLean, Y. Yang, W. Chen, M. Barati: in Advances in Molten Slags, Fluxes, and Salts: Proceedings of The 10th International Conference on Molten Slags, Fluxes and Salts (MOLTEN16), U. Reddy, G. Ramana, P. Chaubal, P.C. Pistorius, U. Pal, eds., 2016, pp. 279-90.

15. C.B. Shi, M.D. Seo, J.W. Cho, and S.H.. Kim: Metall. Mater. Trans. B, 2016, vol. 45B, pp. 1081-97.

16. W. Lu and B.X. Wang: Metall. Mater. Trans. B, 2016, vol. 46B, pp. 852-62.

17. J. Park, P.S. Sridhar, and P.R.J. Fruehan: AISTech - Iron Steel Technol. Conf. Proc., 2012, pp. 2177-84.

18. N. Bessho, H. Yamasaki, T. Fujii, T. Nozaki, and S. Hiwasa: ISIJ Int., 1992, vol. 32, pp. 57-63.

19. S. Spooner, A.N. Assis, J. Warnett, R. Fruehan, and M.A. Williams: 2015, pp. 3-4.

20. J. Park, S. Sridhar, and R.J. Fruehan: Metall. Mater. Trans. B, 2013, vol. 44B, pp. 13-15.

21. J. Park, S. Sridhar, and R.J. Fruehan: Metall. Mater. Trans. B, 2014, vol. 46B, pp. 109-18.

22. M.A. Rhamdhani, G.A. Brooks, and K.S. Coley: Metall. Mater. Trans. B, 2005, vol. 36B, pp. 219-27.

23. A. Sharan and A.W. Cramb: Metall. Mater. Trans. B, 1995, vol. 26B, pp. 87-94.

24. G.A. Brooks, M.A. Rhamdhani, and K.S. Coley: Y. Pan, 2009, vol. 40, pp. 353-62.

25. M.A. Rhamdhani, G.A. Brooks, and K.S. Coley: Metall. Mater. Trans. B, 2005, vol. 36B, pp. 219-27.

26. M.A. Rhamdhani, K.S. Coley, and G.A. Brooks: Metall. Mater. Trans. B, 2005, vol. 36B, pp. 591-604.

27. P.V. Riboud and L.D. Lucas: Can. Metall. Q., 1981, vol. 20 (2), pp. 199-208. https://doi.org/10.1179/cmq.1981.20.2.199.

28. L. Zhang and B.G. Thomas: pp. 1-70.

29. S. Jansson: A Study on Molten Steel/Slag/Refractory Reactions during Ladle Steel Refining, 2005.

30. L. Zhang, S. Taniguchi, and K. Cai: Metall. Mater. Trans. B, 2000, vol. 31B, pp. 253-66.

31. H. Yin, H. Shibata, T. Emi, and M. Suzuki: ISIJ Int., 1997, vol. 37 , pp. $946-55$.

32. H. Yin, H. Shibata, T. Emi, and M. Suzuki: ISIJ Int., 1997, vol. 37 , pp. $936-45$.

Publisher's Note Springer Nature remains neutral with regard to jurisdictional claims in published maps and institutional affiliations. 